\title{
Baroreflex Sensitivity in Premature Infants - Relation to the Parameters Characterizing Intrauterine and Postnatal Condition
}

\author{
K. HASKOVA ${ }^{1}$, M. JAVORKA ${ }^{2,3}$, B. CZIPPELOVA ${ }^{2,3}$, M. ZIBOLEN ${ }^{1}$, K. JAVORKA ${ }^{2,3}$ \\ ${ }^{1}$ Clinic of Neonatology, Jessenius Faculty of Medicine in Martin, Comenius University in \\ Bratislava and University Hospital in Martin, Martin, Slovakia, ${ }^{2}$ Department of Physiology, \\ Jessenius Faculty of Medicine in Martin, Comenius University in Bratislava, Martin, Slovakia, \\ ${ }^{3}$ Biomedical Center Martin, Jessenius Faculty of Medicine in Martin, Comenius University in \\ Bratislava, Martin, Slovakia
}

Received March 17, 2017

Accepted April 21, 2017

\section{Summary}

At present, there are insufficient information about baroreflex sensitivity (BRS) and factors that determine BRS in premature newborns. The objective of this study was to determine the relationship between BRS and the characteristics that reflecting the intrauterine development (gestational age and birth weight), as well as postnatal development (postconception age and the actual weight of the child at the time of measurement). We examined 57 premature infants, who were divided into groups according to gestational age and postconception age as well as birth weight, and weight at the time of measurement. Continuous and noninvasive registration of peripheral blood pressure (BP) was performed in every child within 2-5 min under standard conditions using a Portapres (FMS) device. The results showed a close correlation of baroreflex sensitivity, heart rate and respiratory rate with gestational age, postconception age, birth weight and actual weight at the time of measurement premature newborns. An increase in the characteristics (ages and weights) resulted in increased BRS and diastolic arterial pressure (DAP), and in decreased heart and respiratory rates. Baroreflex sensitivity in the first week was in the group of very premature newborns the lowest $(4.11 \mathrm{~ms} / \mathrm{mmHg}$ ) and in the light premature newborns was almost double $(8.12 \mathrm{~ms} / \mathrm{mmHg})$. BRS increases gradually in relation to postnatal (chronological) and to postconception age as well as to birth and actual weight. The multifactor analysis of BRS identified birth weight and postconception age as the best BRS predictors. The two independent variables together explained $40 \%$ of interindividual BRS variability.

\section{Key words}

Baroreflex sensitivity • Premature newborns

\section{Corresponding author}

K. Javorka, Department of Physiology and Biomedical Center Martin, Jessenius Faculty of Medicine in Martin, Comenius University in Bratislava, Mala Hora 4C, 03601 Martin, Slovakia. E-mail: Javorka@jfmed.uniba.sk

\section{Introduction}

Systemic blood pressure and tissue perfusion in different situations are maintained at physiological levels by the cardiovascular system (CVS) regulation, which starts in intrauterine life and develops during the early postnatal period. The peculiarities of this regulation are determined by morphological and functional status of CVS and the autonomic nervous system (ANS) development.

Baroreflexes are the most important nervous mechanisms regulating the CVS. They play a key role in blood pressure regulation at two levels. They are applied in fast and short-term BP normalization (beat-to-beat), as well as in long-term BP regulation. Activity of baroreceptors leads to reflex responses, including heart rate, cardiac contractility and vascular tone control of resistance and capacitance vessels (e.g. Honzikova and Zavodna 2016).

According to Wood and Tong (1999), baroreflex during intrauterine life has a less physiological 
importance, as evidenced by the fact that chronic sinoaortic denervation does not cause significant changes in foetal BP and its variability.

In the literature is little information about the CVS regulation and baroreflex sensitivity (BRS) in newborns, especially in premature infants and there are missing more studies that assess BRS in premature infants in a comprehensive manner, with anamnestic data (birth weight, weight of the newborn at the time of measurement, gestational and postconception age of the newborn) (Fyfe et al. 2015). This is mainly due to limited methodological procedures for noninvasive continuous blood pressure monitoring in newborns. In neonatological clinical practice, continuous (beat-to-beat) blood pressure monitoring, what is necessary to obtain data on baroreflex sensitivity is almost exclusively used as invasive method with applications limited to certain indications only.

In adults, for non-invasive continuous BP monitoring is used the volume-clamp method (Penaz 1973) by Finometer or Portapres (FMS) devices. Studies of Drouin et al. (1997a), Andriessen et al. (2004) and Yiallorou et al. (2006) showed that this method can also be applied in newborn infants, when modified by deploying a cuff on the child's wrist instead of finger. The relevance and validity of this modified method were verified by comparison with invasive methods (Drouin et al. 1997, Andriessen et al. 2004).

Aim of the study was to determine the baroreflex sensitivity (BRS) in premature newborns and relationships between BRS and parameters reflecting intrauterine (gestational age, birth weight) and early postnatal growth and development (actual weight, postconception age).

\section{Methods}

\section{Subjects}

We examined 57 premature infants, 27 boys and 30 girls of average gestational age $31.8 \pm 3.00$ (25-37) weeks, average postnatal (chronological) age $20.7 \pm 13.5$ (4-56) days, and postconception age 34.4 \pm 1.9 (32-39) weeks. Postconception age was calculated as gestational age + postnatal age in weeks. The average weight of newborns at birth was $1,641 \pm 501 \mathrm{~g}$, and $1,925 \pm 371 \mathrm{~g}$ at the time of measurement. Criteria for inclusion of newborns in the group: children born between 25 and 37 weeks gestation, weighting 1,000-2,500 $\mathrm{g}$ at the time of measurement, wrist circumference $45-75 \mathrm{~mm}$, postnatal age $\geq 4$ days.
The children were in incubators or in heated beds in thermoneutral zones ambient temperature, without signs of respiratory or cardiovascular disorders. They have not medications that could affect their cardiovascular and respiratory systems. During examination, the children were in supine position, they had to be calm and quiet (the child's condition was evaluated according to Stefanski et al. (1984). The child's restlessness belongs to the exclusion criteria. The study was approved by the Ethics Committee of Jessenius Faculty of Medicine, Comenius University in accordance with the Declaration of Helsinki (2000) of the World Medical Association. All parents of the infants enrolled in the study gave their written informed consent prior to the examination.

The infants were divided into the following subgroups by:

Gestational age: Very preterm infants (25-31 weeks gestation) $(n=28)$ and preterm infants (32-37 weeks gestation) $(\mathrm{n}=29)$;

Postconception age: $31-32$ weeks $(\mathrm{n}=10)$, 33-34 weeks $(\mathrm{n}=19), 35-36$ weeks $(\mathrm{n}=21), 37-39$ weeks $(\mathrm{n}=7)$;

Birth weight: less than $1,500 \mathrm{~g} \quad(\mathrm{n}=25)$, 1501-2500 g $(\mathrm{n}=32)$;

Weight at the time of measurement: $1,000-1,500 \mathrm{~g} \quad(\mathrm{n}=10), \quad 1,501-2,000 \mathrm{~g} \quad(\mathrm{n}=20)$, $2,001-2,500 \mathrm{~g}(\mathrm{n}=27)$.

\section{Standardization of measurement conditions}

The children were examined between 8:00 am and 3:00 pm under standard conditions: environment with minimal disturbances - restriction of light and auditory stimuli; constant ambient temperature adjusted to the child's gestational age; loose clothing; examination of the child 1-2 $\mathrm{h}$ after feeding, without dummy to exclude the possible effects of its use (Yiallourou et al. 2014). The children were asleep during the measurement without movements of extremities - behavior pattern 2-3 according to Stefanski et al. (1984).

\section{Instrumentation and software}

For noninvasive and continuous BP measurement was used Portapres device (FMS, Netherlands). Instantaneous beat-to-beat blood pressure and heart/pulse rate values were stored in the device memory and later transferred to a computer and processed by special software (BeatScope, FMS, The Netherlands). From each record, we obtained sections of $200-500$ beat-to-beat 
values of blood pressure and pulse rate visualization and processing of the pressure curve, instantaneous blood pressure and heart rate, as well as the values of mean, systolic and diastolic blood pressure and pulse rate.

\section{Baroreflex sensitivity calculation}

BRS expressed in $\mathrm{ms} / \mathrm{mmHg}$ was assessed using the FMS software BeatScope 1.1a and PRVBRS program by the cross-correlation sequence method. This calculation needs the precise determination of the pulse rate by algorithms applied for measurement of the instant of the begin of the upstroke of the pressure pulse and determination systolic arterial pressure (SAP).

BRS determination using time domain sequential and cross - correlation BRS techniques: BP and PI change must be in the same direction for at least 4 beats. Determination of delays between BP and PI intervals finding of the delay (method of variable delay) with highest cross - correlation over a time span of $12 \mathrm{~s}$. Only if coefficient of determination $\mathrm{r}^{2}$ is $\mathrm{P}=0.01$ BRS was determined. Average value of the BRS for each newborn was calculated from 7-125 BRS values.

\section{Measurement procedure}

Blood pressure measurement was preceded by an appropriate choice of cuff diameter supplied with Portapres $(\mathrm{S} / \mathrm{M} / \mathrm{L})$ corresponding to the wrist circumference of the examined newborn. We measured the wrist circumference, chose the appropriate size of commercially available cuffs, and then we placed the cuff around the right wrist. The child was left at rest for at least $5 \mathrm{~min}$ without inflating the cuff. During each measurement, we recorded the following data in the protocol: child's behavior, oxygen saturation of blood by pulse oximeter (NELLCOR M560 and CAREC APE V100). Oxygen saturation of blood in children ranged from $92.0 \%$ to $100 \%$. Respiratory rate was counted visually, using a stopwatch, at $30 \mathrm{~s}$ intervals. Moreover, we collected and assessed several anamnestic data, such as gestational and postconception age, birth weight and length of the child, weight of child at the time of measurement, etc.

\section{Statistical evaluation}

We used the software SYSTAT 13 for statistical analysis. Within each subgroup of premature infants we used the following tests: ANOVA test or t-test were used in the event that all subgroups had a Gaussian distribution; in the presence of at least one group without a normal distribution of data we used Kruskal-Wallis test, to compare individual groups within the formed subgroups we used post hoc analysis - ANOVA for data with a Gaussian distribution, or Conover-Iman test for data with a non-Gaussian distribution.

To determine the inter-relationship of the assessed parameters of cardiovascular system depending on the clinical history parameters, based on the normality of distribution, we used Pearson correlation test (for parameters with a Gaussian distribution, which included: gestational and postconception age, birth weight, and weight at the time of measurement, values of SAP, DAP and MAP, heart rate, baroreflex sensitivity logarithm) or Spearman correlation test (for parameters without a Gaussian distribution, such as postnatal age and BRS), which was statistically validated by Shapiro-Wilk normality test.

Multiple regression analysis was used to establish mutual prediction of log BRS, as the dependent variable, by independent variables - gestational age, postnatal age, postconception age, birth weight, Apgar score at $10 \mathrm{~min}$, SAP, DAP, heart rate (HR).

$P$ value $<0.05$ was considered statistically significant. The results are presented as arithmetic mean \pm standard deviation (SD), and as median and interquartile range (box and whisker plots).

\section{Results}

\section{Relation between BRS and gestational age}

We found statistically significant differences between evaluated two groups: very premature (Group 1; $25^{\text {th }}-31^{\text {st }}$ gestational week) and premature infants (Group 2; $32^{\text {nd }}-37^{\text {th }}$ g.w.). BRS in the group 1 was $4.1 \pm 1.6 \mathrm{~ms} / \mathrm{mmHg}$, and in the group 2 was $8.1 \pm 5.0 \mathrm{~ms} / \mathrm{mmHg}(\mathrm{P}<0.001)$. Pulse rate and respiratory rate were higher and diastolic blood pressure was lower in the group 1 (Table 1).

\section{Relation between BRS and birth weight}

When comparing the infants with birth weight less than 1,500 $\mathrm{g}$ (Group 1) and those with birth weight from 1,501 to 2,500 $\mathrm{g}$ (Group 2) it was found that group 2 - newborns with higher birth weight had significant higher baroreflex sensitivity (BRS) as well as diastolic pressure (DAP) and lower pulse (PR) and respiratory (RR) rates (Table 2). 
Relation between BRS and postconception age

We also compared the evaluated cardiorespiratory parameters between groups according to postconception age (PCA): Group 1: PCA 31 - 32 weeks; Group 2: PCA 33-34 weeks; Group 3: PCA 35 - 36 weeks; Group 4: PCA 37 - 39 weeks. There were significant differences - increasing by PCA - in BRS among individual groups. The pulse rate (PR) decreased also with increasing postconception age of children. Diastolic and mean blood pressure tended to rise, while respiratory rate tended to drop (Table 3, Fig. 1).

Relation between BRS and actual weight of child at the time measurement

When comparing the evaluated groups (Group 1: newborns weighing 1,000-1,500 g, Group 2: newborns weighing 1,501-2,000 g, and Group 3: newborns weighing 2,001-2,500 g) we found that higher actual weight of the infant corresponds with a significant higher BRS and lower pulse and respiratory rates (Table 4).

Table 1. Baroreflex sensitivity (BRS), systemic blood pressure (systolic - SAP, diastolic - DAP and mean - MAP), pulse rate (PR) and respiratory rate (RR) in the groups of premature infants of different gestational age. The values are expressed as mean \pm standard deviation (SD); ${ }^{*}$ - significant differences: ${ }^{*} \mathrm{P}<0.01,{ }^{* *} \mathrm{P}<0.001$.

\begin{tabular}{lcc}
\hline & Group 1 & Group 2 \\
\hline BRS $(\mathrm{ms} / \mathrm{mmHg})$ & $4.11 \pm 1.59$ & $8.12 \pm 5.00^{* *}$ \\
$S A P(\mathrm{mmHg})$ & $58.70 \pm 8.10$ & $57.70 \pm 6.80$ \\
$D A P(\mathrm{mmHg})$ & $29.70 \pm 9.20$ & $35.0 \pm 60.90^{* *}$ \\
$M A P(\mathrm{mmHg})$ & $40.80 \pm 9.00$ & $44.30 \pm 6.50$ \\
$P R / \mathrm{min}$ & $155.00 \pm 10.20$ & $139.80 \pm 17.30^{* *}$ \\
$R R / \mathrm{min}$ & $55.10 \pm 8.60$ & $49.10 \pm 6.20^{*}$ \\
\hline
\end{tabular}

Table 2. Relation between cardiorespiratory parameters and birth weight. The values are expressed as mean \pm standard deviation (SD). BRS - baroreflex sensitivity, SAP - systolic arterial pressure, DAP - diastolic arterial pressure, MAP - mean arterial pressure, $\mathrm{PR} / \mathrm{min}$ - pulse rate per minute, $\mathrm{RR} / \mathrm{min}$ - respiratory rate per minute, ${ }^{*}$ - significant differences: ${ }^{*} \mathrm{P}<0.01,{ }^{* *} \mathrm{P}<0.001$.

\begin{tabular}{lcc}
\hline Birth weight & Group $\mathbf{1}(<\mathbf{1 , 5 0 0} \mathbf{g})$ & Group 2 (1,501 - 2,500 g) \\
\hline BRS $(\mathrm{ms} / \mathrm{mmHg})$ & $4.4 \pm 2.2$ & $7.0 \pm 4.3^{*}$ \\
SAP $(\mathrm{mmHg})$ & $58.0 \pm 8.2$ & $58.4 \pm 7.0$ \\
$D A P(\mathrm{mmHg})$ & $28.8 \pm 7.5$ & $34.7 \pm 8.2^{*}$ \\
$M A P(\mathrm{mmHg})$ & $40.0 \pm 7.8$ & $44.2 \pm 7.7$ \\
$P R / \mathrm{min}$ & $155.5 \pm 10.8$ & $142.8 \pm 15.3^{* *}$ \\
$R R / \mathrm{min}$ & $56.2 \pm 8.7$ & $48.9 \pm 5.9^{* *}$ \\
\hline
\end{tabular}

Table 3. Dependence of cardiorespiratory parameters on postconception age. The values are expressed as mean \pm standard deviation (SD). SAP - systolic arterial pressure, DAP - diastolic arterial pressure, MAP - mean arterial pressure, PR/min - pulse rate per minute, $\mathrm{RR} / \mathrm{min}$ - respiratory rate per minute, ${ }^{*}$ - significant differences: ${ }^{*} \mathrm{P}<0.01,{ }^{* *} \mathrm{P}<0.001$.

\begin{tabular}{lcccc}
\hline Postconception age (weeks) & $\mathbf{3 1}-\mathbf{3 2}$ & $\mathbf{3 3}-\mathbf{3 4}$ & $\mathbf{3 5}-\mathbf{3 6}$ & $\mathbf{3 7 - 3 9}$ \\
\hline BRS $(\mathrm{ms} / \mathrm{mmHg})$ & $3.28 \pm 1.73$ & $4.90 \pm 1.35^{*}$ & $7.56 \pm 5.34^{*}$ & $9.240 \pm 4.45^{* *}$ \\
$S A P(\mathrm{mmHg})$ & $60.00 \pm 10.40$ & $58.30 \pm 5.00$ & $58.80 \pm 7.80$ & $53.800 \pm 7.20$ \\
$D A P(\mathrm{mmHg})$ & $30.10 \pm 13.10$ & $31.70 \pm 6.50$ & $33.20 \pm 8.30$ & $35.200 \pm 6.20$ \\
$M A P(\mathrm{mmHg})$ & $40.80 \pm 12.90$ & $42.40 \pm 5.40$ & $43.40 \pm 8.00$ & $43.300 \pm 6.08$ \\
$P R / \mathrm{min}$ & $153.90 \pm 9.40$ & $153.30 \pm 11.40$ & $145.80 \pm 14.60$ & $128.000 \pm 23.10^{* *}$ \\
$R R /$ min & $54.70 \pm 5.20$ & $53.70 \pm 7.20$ & $51.10 \pm 9.60$ & $45.625 \pm 3.30$ \\
\hline
\end{tabular}




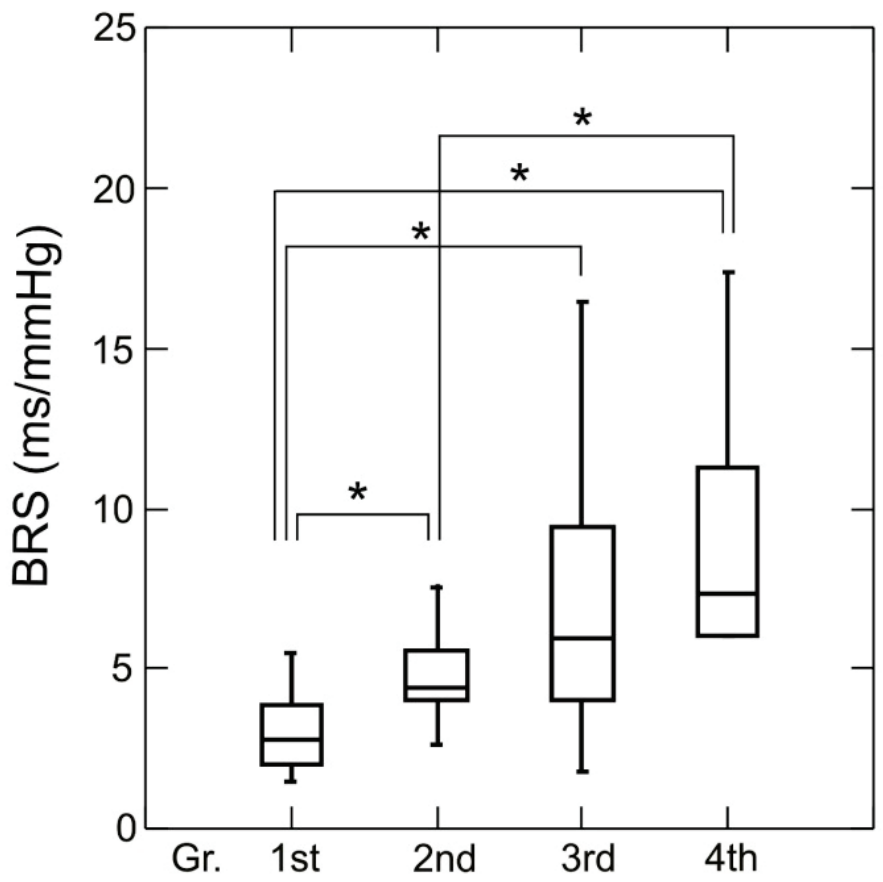

Fig. 1. Baroreflex sensitivity (BRS) according to the postconception age of premature infants. Groups: $1^{\text {st: }} 31-32,2^{\text {nd }}: 33-34,3^{\text {rd }}: 35-36,4^{\text {th }}: 37-39$ weeks. An exact numerical values and of significance of differences are in Table 3.

Table 4. Relation between cardiorespiratory parameters and weight at the time of measurement. The values are expressed as mean \pm standard deviation (SD). BRS - baroreflex sensitivity, SAP - systolic blood pressure, DAP - diastolic arterial pressure, MAP - mean arterial pressure, $\mathrm{PR} / \mathrm{min}$ - pulse rate per minute, $\mathrm{RR} / \mathrm{min}$ - respiratory rate per minute, ${ }^{*}$ - significant differences: ${ }^{*} \mathrm{P}<0.01$, ${ }^{* *} \mathrm{P}<0.001$.

\begin{tabular}{|c|c|c|c|}
\hline Actual weight (g) & $1,000-1,500$ & $1,501-2,000$ & $2,001-2,500$ \\
\hline$B R S(m s / m m H g)$ & $3.8 \pm 1.3$ & $4.7 \pm 3.0$ & $7.3 \pm 4.2 * *$ \\
\hline $\mathrm{SAP}(\mathrm{mm} H g)$ & $61.3 \pm 9.6$ & $59.4 \pm 7.7$ & $56.7 \pm 5.7$ \\
\hline$D A P(m m H g)$ & $31.4 \pm 9.9$ & $30.4 \pm 9.7$ & $34.0 \pm 6.7$ \\
\hline$M A P(m m H g)$ & $43.0 \pm 9.9$ & $41.2 \pm 9.5$ & $43.4 \pm 5.8$ \\
\hline$P R / m i n$ & $161.2 \pm 8.7$ & $148.8 \pm 10.2$ & $143.8 \pm 17.6^{* *}$ \\
\hline$R R / \min$ & $57.8 \pm 6.6$ & $55.3 \pm 8.1$ & $46.6 \pm 5.0^{* *}$ \\
\hline
\end{tabular}

Correlation of BRS and some monitored data across the group of patients

Correlation coefficients in the whole group of premature infants were as follows: BRS: gestational age $\mathrm{R}=0.558$, BRS: postconception age $\mathrm{R}=0.515$, BRS: birth weight $\mathrm{R}=0.543$, and $\mathrm{BRS}$ : actual weight at measurement $\mathrm{R}=0.519$. All of these correlations were highly statistically significant.

\section{Results of BRS assessment by multiple factor analysis}

Multiple factor analysis of BRS was carried out on the entire group of premature infants using three regression models. The first regression model included gestational age, postnatal age, postconception age, birth weight and Apgar score at $10 \mathrm{~min}$ as independent variables, and $\log$ BRS as the dependent (output) variable. Using multistep analysis, the birth weight $(\mathrm{P}=0.001)$ and postconception age $(\mathrm{P}=0.006)$ were identified as the best BRS predictors out of these set of independent variables showing the positive relation with $\log$ BRS, and together explained $40 \%$ of interindividual BRS variability.

The second regression model included, in addition to independent variables from previous model, diastolic (DAP) and systolic (SAP) blood pressure. Multiple factor analysis detected a $56 \%$ association between BRS, postconception age and birth weight as well as $\operatorname{SAP}(\mathrm{P}=0.023)$ and $\operatorname{DAP}(\mathrm{P}<0.001)$, and the independent variables (except SAP) correlated positively with BRS. 
The third regression model included additional independent variable - pulse rate (PR). Multi-step analysis demonstrated $66 \%$ predictability of BRS by postconception age, SAP, DAP and PR. The statistical effect was significant in all independent variables selected for the analysis - DAP and PR $(\mathrm{P}<0.001)$, postconception age $(\mathrm{P}=0.006)$ and $\mathrm{SAP}(\mathrm{P}=0.049)$. The interdependence of SAP and PR towards BRS was negative, while it was positive for DAP and postconception age. The remaining independent variables were not statistically significantly related to the BRS of premature infants.

\section{Discussion}

At present, the incidence of premature births is increasing. Preterm birth has an impact on cardiovascular parameters of preterm infants, as shown by higher heart rate and reduced blood pressure when compared to term born newborns. Furthermore, premature infants can have altered autonomic control of cardiovascular system, which can manifest as abnormalities in heart rate variability and baroreflex (e.g. Fyfe et al. 2014).

In the presented study, the baroreflex sensitivity (BRS) in the premature infants was evaluated by analysis of beat-to-beat blood pressure signals recorded using Portapres (FMS) and by cross-correlation sequence method applied in original software BeatScope 1.1a, PRVBRS program (FMS).

We found significantly lower BRS in group of very preterm neonates with lower gestational age (under 31 weeks) and birth weight (less than 1,500 g) in comparison to light preterm infants. Our results confirm the results of several works studied baroreflex sensitivity in newborns and do not agree with the results of others. Drouin et al. (1997b) have shown that BRS is reduced in preterm compared with term infants. Significantly lower BRS values in premature infants $(3-5 \mathrm{~ms} / \mathrm{mmHg})$ compared to values $(10-15 \mathrm{~ms} / \mathrm{mmHg})$ in full-term newborns were reported by other authors as well (Andriessen et al. 2005, Yiallorou et al. 2010).

It is interesting that the BRS values in preterm infants remained constantly lower than in full-term ones even despite the fact that they developed both with increasing postnatal age. It indicates a slow maturation of BRS with increasing age of prematurely born children. The assumed insufficient maturity of autonomic control of the cardiovascular system, and thus the inadequate baroreflex response (e.g. to hypotension), together with immature respiration regulation, classifies prematurely born children in a risk group prone to sudden infant death syndrome (Matthews 2002).

Delayed maturation of baroreflexes in children born as very premature can be effect of slower extrauterine maturation (Drouin et al. 1997b, Gournay et al. 2002, Yiallorou et al. 2010, Fyfe et al. 2015). We suppose that this slower maturation may be primarily due to mentioned greater immaturity of baroreflex arch and probably also by less stimuli eliciting changes in blood pressure in standard care for very preterm infants, e.g. predominance of lying position in incubators, gentle handling which does not produce changes in blood pressure, etc.

Gournay et al. (2002) found that the BRS values in premature infants were lower, and increased with the growing gestation and postnatal age. Using multiple factor analysis, these authors revealed correlation between gestational age and BRS, but this relationship was not significant. In our group of premature newborns, we found a significant correlation between BRS and gestational age, which accounted, together with birth weight, for nearly $40 \%$ of interindividual BRS variability.

On the other hand, some studies examining the effect of gestational age and/or age after birth on baroreceptor response to tilt or in hypotension have conflicting results. They reported no correlation between gestational age and baroreflex responses (Holden et al. 1985, Golder et al. 2013).

In our study mean value of the BRS in very preterm infants was $4.11 \pm 1.59 \mathrm{~ms} / \mathrm{mmHg}$ and in premature infants was $8.1 \pm 5.0 \mathrm{~ms} / \mathrm{mmHg}$. Comparable value $(8 \pm 5 / 3 \mathrm{~ms} / \mathrm{mmHg})$ was found by Tank et al. (2000) in healthy adults of the age $50-59$ and $60-69$ years and BRS value lower than $3.9 \mathrm{~ms} / \mathrm{mmHg}$ was found also in $5 \%$ of healthy adolescent subjects (Honzikova and Fiser 2009). Honzikova and Zavodna (2016) hypothesize that low BRS can play an active role in the hypertension in youth. This value approaches also to the critical value for the risk of sudden cardiac death in patients after myocardial infarction and corresponds to the value present in adult hypertensive patients. However, situation in very premature infants is different to the situation in adolescents and adults. BRS values in early postnatal life in premature infants probably are in close correlation to BRS values during intrauterine life (tracking phenomenon), when baroreflexes do not play crucial vital important role. The baroreflexes are more important in 
later postnatal life when there are more situations (change in position, food intake, external stimuli) eliciting changes in blood pressure as well as in perfusion (e.g. cerebral circulation), and these changes should be very quickly normalized to appropriate physiological values. This situations and „training of baroreflexes“ together with a maturation of the ANS and control mechanisms can have a reflection on the development of cardiovascular control and on correlations between BRS and postconception age. BRS increases gradually to cover greater demands on baroreflex control of CVS.

The findings of the BRS increase and pulse rate decrease depending on rising postconception age (gestational plus postnatal age) in our group of premature infants are in agreement with the results of the work by Andriessen et al. (2005). These authors studied heart rate variability, systolic blood pressure (SAP), as well as baroreflex sensitivity depending on postconception age in a group of clinically stable newborns. They found a significant BRS increase with elevated values in spectral total power (TP) as well as in all bands of power spectra (LF, HF). Heart rate variability (HRV), the average inter beats intervals and SAP values grew significantly. The authors suggest that an increase in fluctuations of $\mathrm{HR}$ and increase in BRS with postmenstrual age are related to the maturation of the vagal system.

The multi-factor analysis in our study found a $56 \%$ association between BRS and postconception age, birth weight, as well as SAP and DAP, where their correlation was positive in exception of SAP. The combination of SAP, DAP, HR and postconception age represented variables with the highest BRS prediction in premature newborns, namely $66 \%$, where the SAP and PR interdependence towards BRS was negative, while with DAP and postconception age it was positive.

Lower baroreflex sensitivity and increased heart rate in more premature newborns indicates a lower level of cardiovascular system regulation by the autonomic nervous system. Although autonomic innervation of the sympathetic and parasympathetic system is already present at birth, it is still under the dominant influence of its sympathetic component at low resting vagal tone, which is manifested by a higher heart rate, especially in premature newborns. Immaturity may also be manifested in baroreflex function, especially due to the underdeveloped central and efferent part of the reflex arc at birth. Therefore, baroreflex sensitivity matures with increasing gestational, as well as postnatal and postconception age of the child.

\section{Conclusions}

The results confirm and extend the knowledge of the baroreflex sensitivity in premature infants as well as about its relation to birth weight, gestational, postnatal and postconception age in premature newborns. The findings showed lower BRS and diastolic arterial pressure (DAP) and higher pulse and respiratory rates in very premature infants in comparison to light premature ones, and a close correlation of baroreflex sensitivity, diastolic blood pressure, pulse rate and respiratory rate with gestational age, postconception age, birth weight and actual weight at the time of measurement premature newborns. Baroreflex sensitivity in the first postnatal week was in the group of very premature newborns the lowest $(4.11 \mathrm{~ms} / \mathrm{mmHg})$ and in the light premature newborns was almost double $(8.12 \mathrm{~ms} / \mathrm{mmHg})$. The multifactor analysis of BRS identified birth weight and postconception age as the best BRS predictors. The two independent variables together explained $40 \%$ of interindividual BRS variability.

\section{Conflict of Interest}

There is no conflict of interest.

\section{Acknowledgements}

This work was supported by Projects: VEGA No. 1/0202/16, APVV - 0235-12, "Biomedical Center Martin" ITMS Code 26220220187 and by the project Center of Excellence of Perinatology Research (CEPV).

\section{References}

ANDRIESSEN P, SCHOFFELEN RL, BERENDSEN RC, DE BEER NA, OEI SG, WIJN PF, BLANCO CE: Noninvasive assessment of blood pressure variability in preterm infants. Pediatr Res 55: 220-223, 2004.

ANDRIESSEN P, OETOMO SB, PETERS C, VERMEULEN B, WIJN PF, BLANCO CE: Baroreceptor reflex sensitivity in human neonates: The effect of postmenstrual age. $J$ Physiol 568: 333-341, 2005. 
DROUIN E, GOURNAY V, CALAMEL J, MOUZARD A, ROZE JC: Feasibility of using finger arterial pressure in neonates. Arch Dis Child Fetal Neonatal Ed 77: F139-F140, 1997a.

DROUIN E, GOURNAY V, CALAMEL J, MOUZARD A, ROZE JC: Assessment of spontaneous baroreflex sensitivity in neonates. Arch Dis Child Fetal Neonatal Ed 76: F108-F112, $1997 \mathrm{~b}$.

FYFE KL, YIALLOUROU SR, WONG FY, HORNE RS: The development of cardiovascular and cerebral vascular control in preterm infants. Sleep Med Rev 18: 299-310, 2014.

FYFE KL, YIALLOUROU SR, WONG FY, ODOI A, WALKER AM, HORNE RS: Gestational age at birth affects maturation of baroreflex control. J Pediat 166: 559-565, 2015.

GOLDER V, HEPPONSTALL M, YIALLOUROU SR, ODOI A, HORNE RS: Autonomic cardiovascular control in hypotensive critically ill preterm infants is impaired during the first days of life. Early Hum Dev 89: 419-423, 2013.;

GOURNAY V, DROUIN E, ROZE JC: Development of baroreflex control of heart rate in preterm and full term infants. Arch Dis Child Fetal Neonatal Ed 86: F151-F154, 2002.

HOLDEN K, MORGAN JS, KRAUSS AN, AULD PA: Incomplete baroreceptor responses in newborn infants. $A m$ J Perinatol 2: 31-34, 1985.

HONZIKOVA N, FISER B: Baroreflex sensitivity and essential hypertension in adolescents. Physiol Res 58: 605-612, 2009.

HONZIKOVA N, ZAVODNA E: Baroreflex sensitivity in children and adolescents: physiology, hypertension, obesity, diabetes mellitus. Physiol Res 65: 879-889, 2016.

MATTHEWS T: Sudden infant death syndrome: A defect in circulatory control? Child Care Health Dev 28: 41-43, 2002.

PENAZ J: Photoelectric measurement of blood pressure, volume and flow in the finger. Dresden. Digest 10th Int Conf Med Biol Engng 2: 104, 1973.

STEFANSKI M, SCHULZE K, BATEMAN D, KAIRAM R, PEDLEY TA, MASTERSON J, JAMES LS: A scoring system for states of sleep and wakefulness in term and preterm infants. Pediat Res 18: 58-62, 1984.

TANK J, BAEVSKI RM, FENDER A, BAEVSKI A, GRAVES KF, PLOEWKA K, WECK M: Reference values of indices of spontaneous baroreceptor reflex sensitivity. Am J Hypertens 13: 268-275, 2000.

WOOD CH, TONG H: Central nervous system regulation of reflex responses to hypotension during fetal life. $\mathrm{Am}$ J Physiol Regul Integr Comp Physiol 227: R1541-R1552, 1999.

YIALLOUROU SR, WALKER AM, HORNE RS: Validation of a new noninvasive method to measure blood pressure and assess baroreflex sensitivity in preterm infants during sleep. Sleep 29: 1083-1088, 2006.

YIALLOUROU SR, SANDS SA, WALKER AM, HORNE RS: Postnatal development of baroreflex sensitivity in infancy. $J$ Physiol 588: 2193-2203, 2010.

YIALLOUROU SR, POOLE H, PRATHIVADI P, ODOI A, WONG FY, HORNE RS: The effects of dummy/pacifier use on infant blood pressure and autonomic activity during sleep. Sleep Med 15: 1508-1516, 2014. 